ARTICLE

Received 24 Jun 2014 | Accepted 13 Jan 2015 | Published 19 Feb $2015 \quad$ DOl: 10.1038/ncomms7278

\title{
Biophysical mechanisms that maintain biodiversity through trade-offs
}

Justin R. Meyer', Ivana Gudelj² \& Robert Beardmore²

Trade-offs are thought to arise from inevitable, biophysical limitations that prevent organisms from optimizing multiple traits simultaneously. A leading explanation for biodiversity maintenance is a theory that if the shape, or geometry, of a trade-off is right, then multiple species can coexist. Testing this theory, however, is difficult as trait data is usually too noisy to discern shape, or trade-offs necessary for the theory are not observed in vivo. To address this, we infer geometry directly from the biophysical mechanisms that cause trade-offs, deriving the geometry of two by studying nutrient uptake and metabolic properties common to all living cells. To test for their presence in vivo we isolated Escherichia coli mutants that vary in a nutrient transporter, LamB, and found evidence for both trade-offs. Consistent with data, population genetics models incorporating the trade-offs successfully predict the co-maintenance of three distinct genetic lineages, demonstrating that trade-off geometry can be deduced from fundamental principles of living cells and used to predict stable genetic polymorphisms.

\footnotetext{
${ }^{1}$ Division of Biology, Section in Ecology, Behavior and Evolution, University of California, San Diego, La Jolla, California 92093, USA. ${ }^{2}$ Biosciences, University of Exeter, Geoffrey Pope Building, Stocker Road, Exeter EX4 4QD, UK. Correspondence and requests for materials should be addressed to J.R.M. (email: jrmeyer@ucsd.edu) or to R.B. (email: r.e.beardmore@exeter.ac.uk).
} 
$\mathrm{T}$ rade-offs between life-history traits are seen as a key part of the processes that maintain the rich biodiversity observed in microbes ${ }^{1}$, plants ${ }^{2}$, insects ${ }^{3}$ and rain forests ${ }^{4}$. Due to its importance to biodiversity theory, a search for trade-off data has become the subject of a burgeoning experimental literature using microbial populations in the laboratory ${ }^{1,5-12}$. However, despite the significance of trade-off geometry to the theory and the longpostulated hypothesis that trade-offs arise from life-history constraints, we know of no successful derivation of trade-off geometry in living systems from fundamental physical or biochemical principles. Resolving this absence is critical if we are to successfuly predict conditions that maintain diverse microbial communities. Here we present a mechanistic theory, supported by data, on the geometry of two trade-offs among microbes: one is a feeding trade-off, one is a growth trade-off. These trade-offs have been studied before but their shapes have remained elusive, yet here we find agreement between theory and data showing that one trade-off is convex and the other is sigmoidal and both are implicated in diversity maintenance.

The first trade-off we study is the metabolic rate-yield trade-off (the RYTO or 'waste from haste' trade-off ${ }^{13,14}$ ) whose shape we found by culturing Escherichia coli at different growth rates. The second trade-off shape we deduce is that of the nutrient uptake rate-affinity trade-off (the RATO ${ }^{14,15}$ ) by measuring the growth kinetics of a library of mutant E. coli that varied in a sugar transporter, LamB. Given these two geometries, prior theory predicts that there could be nutrient conditions where divergent consumer strategies would coexist in a microbial population ${ }^{16}$. To test this, we used bespoke theoretical population genetics models to determine environmental conditions that would support a multiplicity of nutrient transporter genotypes in E. coli. However, this theory successfully predicted the frequency dynamics of three genetic lineages in a laboratory microcosm only when it incorporated two trade-offs, not one. We therefore establish that trade-offs are a consequence of the physical necessities of life and moreover genetic polymorphisms and biodiversity patterns can be predicted from the resulting trade-off geometries.

\section{Results}

Many mechanisms have been proposed for the RYTO in bacteria, both physiological ${ }^{17}$ and metabolic ${ }^{13}$, and a quadratic shape is thought to describe $\mathrm{it}^{13}$. Attempts to find the RYTO in vivo, however, have been mixed. A negative correlation between growth rate and yield has been observed but without the quadratic form ${ }^{12}$. Moreover, positive rate-yield correlations, the antithesis of a rate-yield trade-off, have been observed in other bacterial species ${ }^{9}$ and, curiously, this relationship is also predicted to be constrained by a quadratic geometry ${ }^{10}$. We can reconcile these apparent contradictions by following a suggestion ${ }^{9}$ that $r-k$ relationships can be manipulated by varying the carbon richness of the environment in which bacteria grow (for clarity, we use $k$ for carrying capacity where others use $K$ ). We hypothesize that $r$ and $k$ are indeed positively correlated and that the shape of the RYTO can be extracted from $r-k$ data sets.

To study the RYTO, we cultured E. coli B (REL606) in Davis minimal (DM) medium supplemented with a range of sugar (maltotriose) concentrations. We determined their $r$ (exponential growth rate) and $k$ (maximal population size) values by fitting the mathematical logistic model $\frac{\mathrm{d}}{\mathrm{d} t} x=r x(1-x / k)$ to growth data, where $x(t)$ denotes bacterial population density at time $t$ (Supplementary Discussion, section C). At low sugar concentrations, ranging from 1 to $8 \mu \mathrm{g} \mathrm{ml}^{-1}$ the experiment recapitulates a prior data set ${ }^{9}$, whereby a positive, linear correlation between $r$ and $k$ is observed (Fig. 1a). However, when observations from higher maltotriose concentrations are added to the data, ranging from 1 to $125 \mu \mathrm{g} \mathrm{ml}^{-1}$, the $r-k$ relationship becomes nonlinear because $r$ saturates. This is expected because growth rate is limited, whereas $k$ increases proportionally to the maltotriose supplied (Fig. 1a, Supplementary Fig. 15). Although $r$ and $k$ adaptation are classically viewed as conflicting life-history strategies ${ }^{18}$ here they are positively correlated. Nevertheless, this is still consistent with a RYTO, as we now explain.

Assume, for the moment, that $\mathrm{c}$ is a constant representing the biomass yield of $E$. coli per maltotriose, when cultured with maltotriose as the sole carbon source at supply concentration $S_{0} \mu \mathrm{g} \mathrm{ml}^{-1}$. We assume Monod growth kinetics, so the bacterial population density $B$ changes as $\frac{\mathrm{d}}{\mathrm{d} t} B=\mathrm{c} B V_{\max } S /\left(K_{\mathrm{m}}+S\right)$ and maltotriose reduces in concentration as $\frac{\mathrm{d}}{\mathrm{d} t} S=-B V_{\max } S /$ $\left(K_{\mathrm{m}}+S\right)$, where $V_{\max }$ is the maximal rate of uptake of maltotriose and $K_{\mathrm{m}}$ is the maltotriose half saturation constant. The initial condition $S(0)=S_{0}$ applies here, $B(0)$ represents a bacterial inoculate whose size is assumed negligibly small.

A conservation law holds in this model, $B(t) / \mathrm{c}+S(t)=B(0) /$ $\mathrm{c}+S_{0}$, so that when $S \approx 0$ at carrying capacity, $k=B \approx \mathrm{c} \cdot S_{0}$ thus $k$ is proportional to the maltoriose supplied, if we neglect $B(0)$. If we approximate exponential growth using the Monod model, so $r \approx c V_{\max } S_{0} /\left(K_{\mathrm{m}}+S_{0}\right)$, then $k / r \approx\left(K_{\mathrm{m}}+S_{0}\right) / V_{\max }$ and so $k$ and $k / r$ should both exhibit linear dependence on $S_{0}$, hence $k$ and $r$ should be positively correlated. This is consistent with our observations (for $k$ versus $S_{0}: R^{2}>0.99, P<10^{-8}$; for $k / r$ versus $S_{0}: R^{2} \approx 0.93, \quad P<10^{-4}$; Supplementary Fig. 15). However, observations also indicate that yield, meaning $\mathrm{c}$ or $k / S_{0}$, is not constant because it decreases with increasing maltotriose supply (Fig. 1b, $F$-test versus constant model, $F=17.3, P<0.00014$ ).

We therefore need a more accurate description of the variableyield data in Fig. 1c. So, we relax our assumption that yield is a constant independent of the carbon supplied. Maltotriose import and metabolism is carried out by the genes of the mal regulon ${ }^{19}$. This set is induced by malT, which itself is expressed when maltodextrins like maltotriose are present. The uptake of maltodextrins is facilitated by the LamB maltoporin in combination with the periplasmic maltodextrin-binding protein MalE and the ATP-driven inner-membrane transport system $\mathrm{MalGFK}_{2}$. Once in the cytoplasm, maltotriose is degraded by MalZ into maltose and glucose, MalQ then produces two glucoses for each available maltose. Given this, a theoretical form for the rate and yield of maltose metabolism can be derived (Supplementary Discussion, section B) by representing glycolysis and the tricarboxylic acid (TCA) cycle as a branched pathway ${ }^{20}$ (Fig. 1a). Using ATP yield as a proxy for biomass yield, this model predicts a relationship between carbon supply, $S_{0}$, and cell yield. Writing $c\left(S_{0}\right)$ to represent this relationship, it has the following repression-activation form

$$
c\left(S_{0}\right)=c_{\mathrm{hi}} \frac{1}{1+p S_{0}}+\frac{p S_{0}}{1+p S_{0}} c_{\mathrm{lo}} .
$$

Here $c_{\mathrm{hi}}$ represents the highest yield attainable, achieved at the lowest sugar concentrations, whereas $c_{\mathrm{lo}}$ is the yield attained when sugar is abundant, $p$ is a phenotype that controls the rate of decrease in yield with increasing sugar supply.

The positive $r-k$ correlation predicted by the constant and variable-yield assumptions are consistent with $r-k$ data (Fig. 1b) with agreement across 2 orders of magnitude of maltotriose supply in both cases. However, only the decreasing yield model correctly captures the yield data across the full range of maltotriose concentrations (Fig. 1c, $R^{2}>0.99, P \ll 10^{-15}$ from above $F$-test). The resulting five-parameter, within-strain geometric RYTO model

$$
\text { (growth rate, cell yield })=\left(c\left(S_{0}\right) \frac{S_{0} V_{\max }}{K_{\mathrm{m}}+S_{0}}, c\left(S_{0}\right)\right)
$$




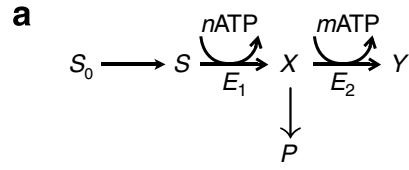

b

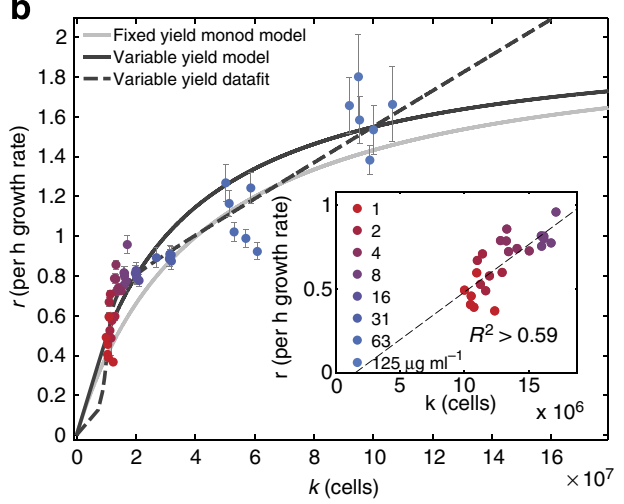

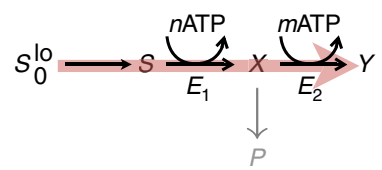

C

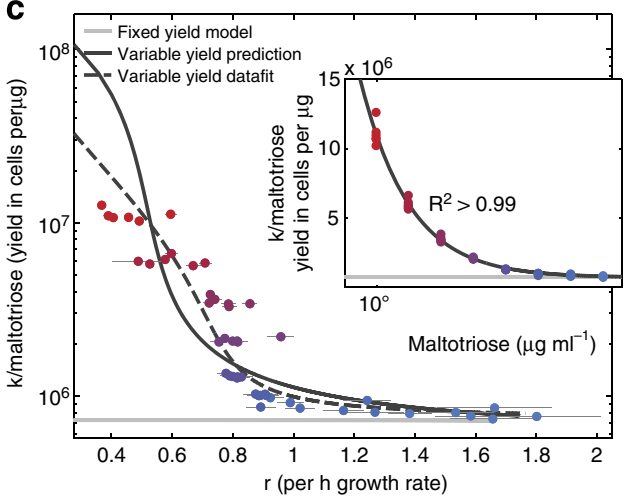

Figure 1 | The RYTO shape can be derived from a simple branched metabolic pathway model and subsequently found in bacterial growth data. (a) A branched pathway predicts that cell yield, c, can be written in the form of equation (1) (derivation in Supplementary Discussion, section B). (b) As $S_{0}$ increases to $125 \mathrm{~g} \mathrm{ml}^{-1}, r$ begins to saturate, whereas $k$ continues to increase. Moreover, two theoretical Monod models (light and dark grey) assuming, respectively, fixed and variable yield are both compatible with this $(k, r)$ data set. (b inset) When $S_{0}$ ranges from 1 to $8 \mu \mathrm{g} \mathrm{ml}{ }^{-1}, r$ and $k$ appear linearly correlated, an observation consistent with published data 9 (error bars indicate mean \pm s.e.m., horizontal s.e.m. bars are present but cannot be seen, six replicates per maltotriose concentration). (c) The variable-yield model (Equation 2) was used to estimate rate-yield trade-off geometry: the solid line shows the predicted geometry using the data set from (b), the dashed line improves on this prediction by fitting equation (2) directly to that data set (Supplementary Table 2 contains relevant parameter estimates). Both of these trade-off models are sigmoidal, note, and the resulting predicted growth rate is shown as a dashed line in $\mathbf{b}$. (c inset) A constant yield assumption fails when yield is plotted versus maltotriose concentration (light grey line). The nonlinear yield form from equation $(1), c\left(S_{0}\right)$, correctly captures the data (dark grey line).

has a sigmoidal shape that compares favourably with data (Fig. 1c). Note that equation (2) has only four independent parameters, $V_{\max }$ merely converts cell yield into a rate.

The logistic equation contains no explicit term for nutrient availability so, we deployed the following improvement to that model that better captures different bacterial growth phases and which can also represent $N$-strain, frequency- and densitydependent competition for maltotriose (where $N \geq 1$ ):

$$
\begin{aligned}
\frac{\mathrm{d}}{\mathrm{d} t} B_{i} & =v n_{i}+G_{i}(S) B_{i}, \quad \frac{\mathrm{d}}{\mathrm{d} t} S=-\sum_{i=1}^{N} U_{i}(S) B_{i}, \\
\frac{\mathrm{d}}{\mathrm{d} t} n_{i} & =-v n_{i} .
\end{aligned}
$$

Here $B_{i}$ is density of the $i$-th strain, growth rate is $G_{i}(S)=$ $c_{i} \cdot U_{i}(S), c_{i}$ is cell yield and $U_{i}(S)$ is nutrient uptake rate (at nutrient concentration $S$ ):

$$
U_{i}(S):=\frac{V_{\max }^{i} S}{K_{m}^{i}+S}
$$

The variable $n_{i}$ represents a subpopulation of the inoculate that may be in a non-vegetative state and the associated $v_{i}$-dependent terms allow the model to capture the transition from lag to exponential phase. When $N=1$ equation (3) is consistent with the RYTO data for E. coli strain REL606 (Supplementary Fig. 14) and when $N>1$, it can predict the outcome of multi-strain competition experiments (Supplementary Discussion, section C).

Pursuing evidence of a between-strain RYTO, we generated a library of E. coli mutants that vary in growth rate because of mutations that interfere with maltotriose uptake. E. coli uses LamB to transport maltotriose across the outer membrane, however, it may also be taken up by $\mathrm{OmpF}$, albeit over an order of magnitude more slowly ${ }^{21,22}$. LamB is also a receptor for the virus $\lambda$, which provides a convent way to generate LamB mutations.
Previous work has shown that when E. coli and $\lambda$ are co-cultured, E. coli rapidly evolves resistance through an array of $\operatorname{lam} B$ mutations $^{23,24}$, some of which alter the diffusion of maltodextrins across the outer membrane by altering the pore dimensions ${ }^{25}$. We therefore co-cultured 100 separate populations in batch for 3 days and isolated 2 bacteria per population. The large number of populations and low sampling intensity within each population helped maximize the number of unique lamB mutants isolated, the short time period ensured only a single mutation occurred in the $\operatorname{lam} B$ gene and nowhere else in the genome. Altogether, we harvested 50 novel E. coli strains with SNPs, insertions and deletions in the lamB gene, the library also includes frameshift mutations that render LamB inoperable (Supplementary Table 1).

We estimated uptake rate, yield and therefore growth rate for each strain by culturing them separately in DM media supplemented with 10,25 and $250 \mu \mathrm{g} \mathrm{ml}^{-1}$ maltotriose by fitting equation (3) to that data (Supplementary Discussion, section C). This consistently produced similar values for yield between strains and environments and significant evidence for a negative rate-yield correlation between strains was observed but only at a maltotriose concentration of $250 \mu \mathrm{g} \mathrm{ml}^{-1}$, not at $25 \mu \mathrm{g} \mathrm{ml}^{-1}$ $(P<0.00064$ and $P>0.16$, respectively; see Fig. $2 \mathrm{a})$.

The same data set points to a second possible trade-off: a correlation between $K_{\mathrm{m}}$ and $V_{\max }$ (Fig. $3 \mathrm{a}, R^{2} \approx 0.58, F \approx 70.5$, $\left.P<10^{-10}\right)$. This prediction is consistent with prior single transporter models for small-molecule uptake, which show that changes to a transporter's affinity for the molecule will produce concomitant changes in its maximal translocation rate ${ }^{26-28}$. This was demonstrated by distorting the performance of transmembrane channels by creating potential energy wells within a synthetic pore using holographic tweezers. These experiments showed that an optimal transporter configuration must balance a high translocation probability and 'not too long' a blockage of the channel ${ }^{27}$; this is the physical basis of the RATO. 

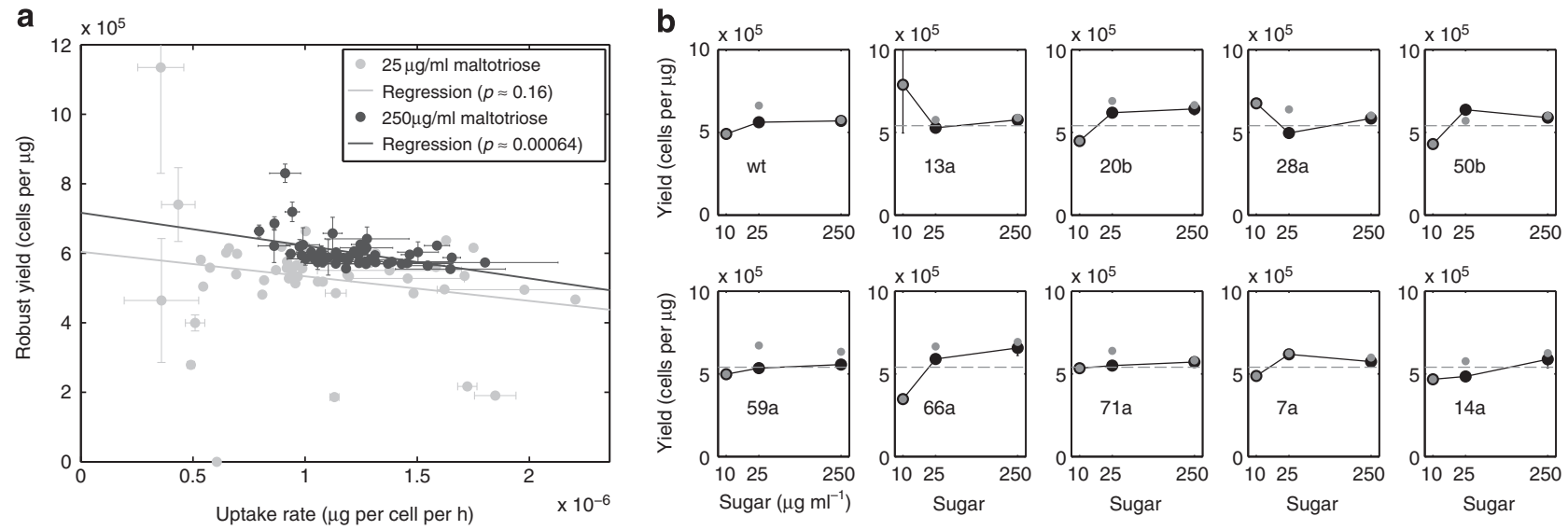

Figure 2 | The evidence for a between-strain RYTO is not significant at all maltotriose concentrations tested. (a) Estimated maltotriose uptake rate versus a robust estimate of cell yield (Supplementary Discussion, section C) for every genotype in the lamB mutant library at two different maltotriose supply concentrations $\left(S_{0}=25\right.$ and $250 \mu \mathrm{g} \mathrm{ml}^{-1}$ ). Linear regressions show evidence of a between-strain negative correlation in yield but only when $S_{0}=250$ (error bars indicate mean \pm s.d., $n=8$; regression: $R^{2} \approx 0.21$, F-statistic versus constant model: $13.3, P<0.00064$ ). (b) Ten strains from the library exhibit a feature common to all: although there are fluctuations due to experimental variation, cell yield is estimated consistently between strains across a range of maltotriose concentrations (error bars indicate mean \pm s.e.m., $n=3$; black and grey dots represent two different yield estimation algorithms, the horizontal dashed line is mean wild-type yield).
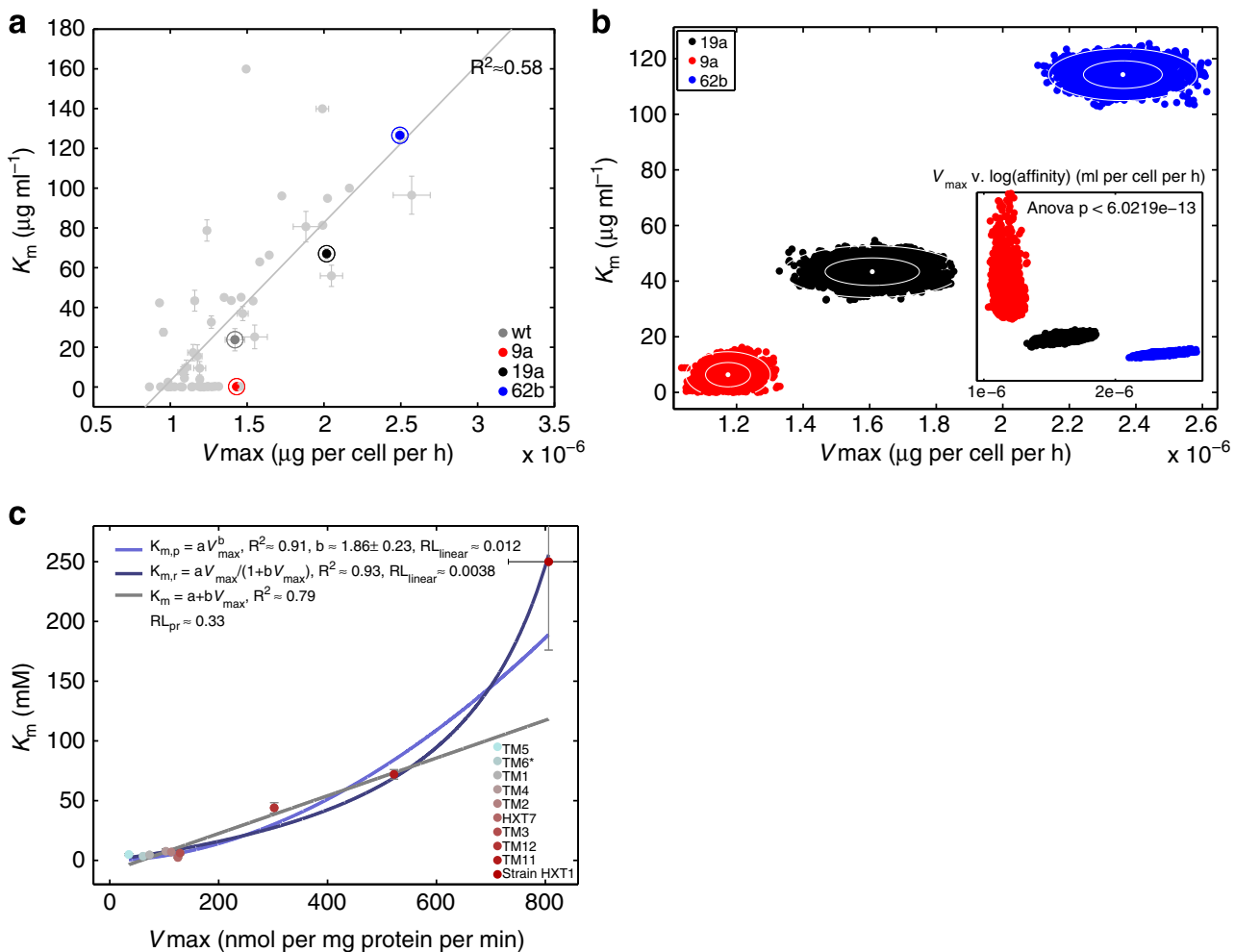

Figure 3 | RATO data in the E. coli library is too noisy to discern a nonlinear shape, however, a prior Saccharomyces cerevisiae data set has a nonlinear RATO. (a) An indication that the $E$. coli library is consistent with a between-strain RATO: estimated values of $K_{\mathrm{m}}$ and $V_{\text {max }}$ are positively correlated (error bars indicate mean \pm s.e.m., $n=8$ ). (b) Maximum likelihood estimates of $\left(K_{m}, V_{\max }\right)$ for library strains $9 a$, 19 a and $62 \mathrm{~b}$ showing $\sim 70$ and $95 \%$ confidence contours of marginal posterior distributions determined using the Matlab MCMC Toolbox (MLEs of $K_{\mathrm{m}}$ : $0.011 \mathrm{mM}, 0.080 \mathrm{mM}$ and $0.211 \mathrm{mM}$, respectively. As a comparison, E. coli strains from the literature ${ }^{38}$ have values $\sim 0.4 \mathrm{mM}$ ). (b inset) Using this data, library strains were isolated ( $9 a$, $19 a$ and $62 \mathrm{~b}$ ) that exhibit a between-strain RATO (see analysis of variance in main text). (c) A whole cell S. cerevisiae $\left(K_{\mathrm{m}}, V_{\max }\right)$ data set (mean data and standard error bars from ref. 26) exhibits a nonlinear uptake RATO. Two robust geometric data fits are shown next to the robust linear regression $K_{\mathrm{m}}=a+b V_{\text {max }}$ : a near-quadratic power law, $K_{\mathrm{m}, \mathrm{p}}$ and a rational function $K_{\mathrm{m}, \mathrm{r}}$. Relative likelihoods of the two nonlinear fits indicate both are significantly better descriptors of the data than a linear function (the indicated $\mathrm{RL}_{\text {linear }}$ values are the relative likelihoods for each fit). This demonstrates consistency between the predicted nonlinear RATO shape (equation 5) and data.

Different theories are possible for RATO geometries depending on the assumptions they use. A linear relationship between $K_{\mathrm{m}}$ and $V_{\max }$ can be derived ${ }^{27}$ (as is done in equation (6) of the latter reference), whereby $K_{\mathrm{m}}=p V_{\max }+q$ for parameters $(p, q)$. This result is consistent with a theoretical enzymatic model based on a transporter embedded within a single membrane 
a

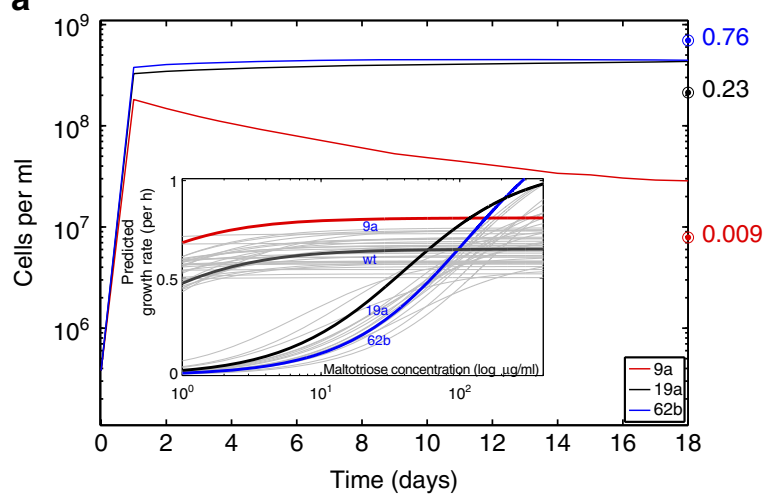

C

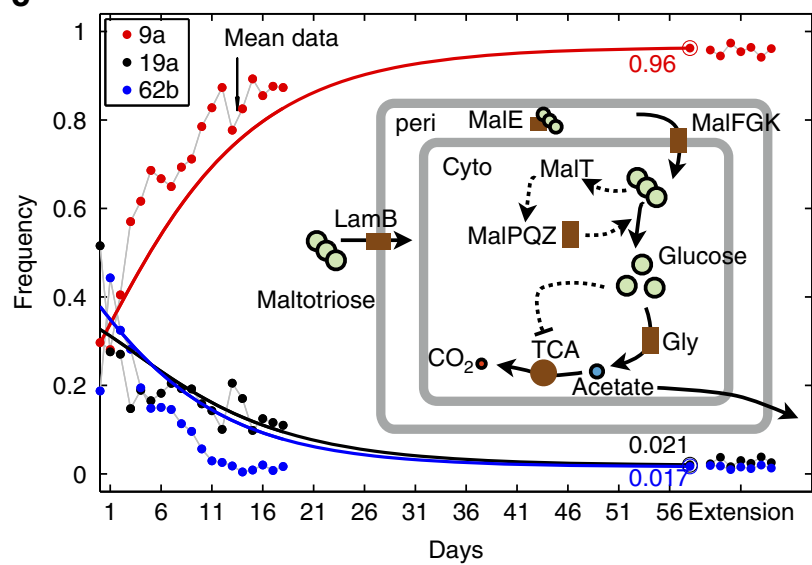

b

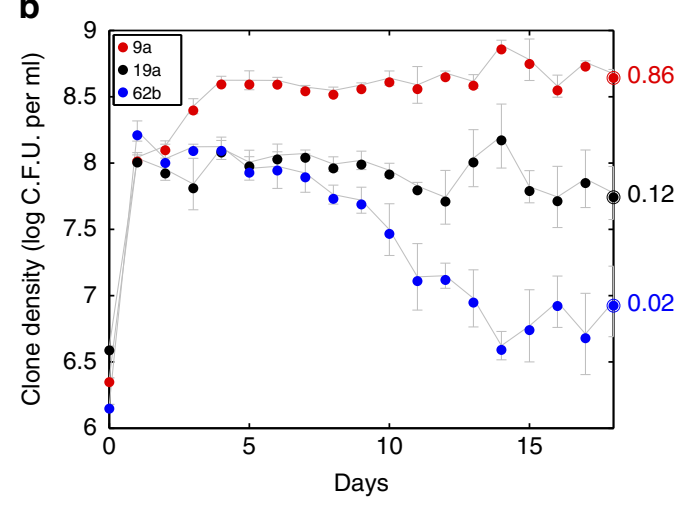

d

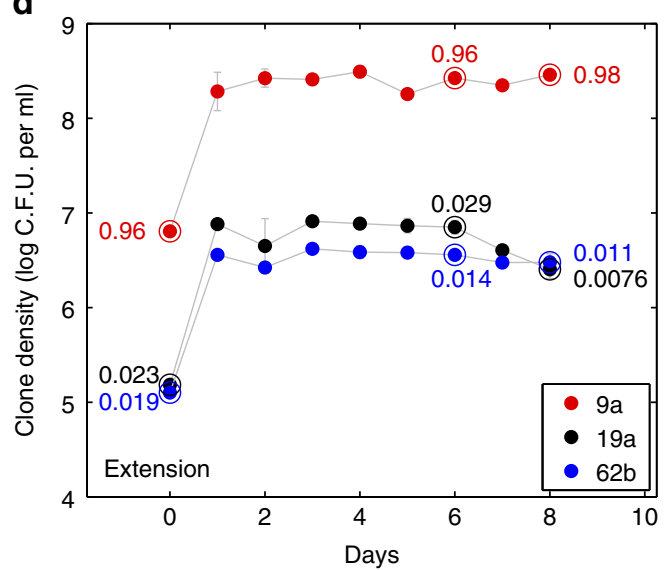

Figure 4 | As predicted by population genetics theory, three lamB mutant strains can be stably co-maintained in a laboratory microcosm.

(a) A theoretical three-strain co-culture model ( $N=3$ in a seasonal extension of equation 3 ) containing a RATO but no RYTO predicts strain dynamics for 18 days when maltotriose is supplied each day at $300 \mu \mathrm{g} \mathrm{ml}{ }^{-1}$. Maltotriose uptake rates, affinities and yields were estimated for each strain in monoculture and the inset shows estimated growth rate for every library strain: strains $9 a, 19 a$ and $62 b$ are predicted to have fastest growth in different maltotriose concentration windows. (b) While the model correctly predicts the co-maintenance of three strains, this was not at the strain frequencies that were subsequently observed. Note, $62 \mathrm{~b}$ has mean population size on day 18 of over one million cells (colony-forming units) despite a mean relative frequency of $2.4 \%$ (error bars indicate mean \pm s.e.m., $n=3$ ). (c) A RYTO was then introduced into the model that was used to make the predictions in a and the resulting model is consistent with strain frequencies (Supplementary Discussion, section D). One model simulation is shown (thick lines) superimposed on observed mean strain frequencies (dots). (c inset) The internal complexity of the E. coli strains on which simulations in c are based, assuming only polymorphisms in lamB. (d) The three strains were co-cultured anew in the same conditions but this time they were inoculated at the model-predicted steady state frequencies, these frequencies were then maintained for a period of 7 days (Supplementary Discussion, section D). Mean observed frequencies are shown next to the model predictions in $\mathbf{c}$ by the label 'extension', the observed densities of this extension are shown in $\mathbf{d}$. Note: numerical text labels in each plot indicate relative frequencies of each strain.

(Supplementary Discussion, section B), so this would appear to be the description of the trade-off we seek. However, affinity for a carbon source, $a$, is usually defined by $a:=\frac{\mathrm{d} U}{\mathrm{~d} S}(0)=V_{\max } / K_{\mathrm{m}}$ and so strains with different $V_{\max }$ and $K_{\mathrm{m}}$ phenotypes could have a common maltotriose affinity, if $q=0$. However, RATO data observed between the E. coli library strains is too noisy to eliminate linearity or to resolve shape (Fig. 3a). To remedy this, we used a related Saccharomyces cerevisiae data set where glucose transporters had been genetically manipulated ${ }^{26}$. As this data exhibits significant nonlinearity (see Fig. $3 \mathrm{~b}$ for the relative likelihoods, $\mathrm{RL}_{\text {linear }}$, of linear and nonlinear data fits where $\mathrm{RL}_{\text {linear }}<0.013$ ), the prior argument supporting a linear $K_{\mathrm{m}}-V_{\text {max }}$ relationship will thus have to be modified.

On reflection, small-molecule uptake in some microbes is a two-step process: entry into the cytoplasm of Gram-negative bacteria and yeast requires the passage through two diffusive barriers, not one. Modifying the above single membrane model to account for this, $K_{\mathrm{m}}$ depends nonlinearly on $V_{\max }$ in the resulting theory (Supplementary Discussion, section B):

$$
K_{\mathrm{m}} \approx \frac{p V_{\max }^{2}+q V_{\max }+r}{1+s V_{\max }},
$$

where $(p, q, r, s)$ are parameters. A quadratic nonlinearity here is expected because if transport through one diffusive barrier leads to a linear $K_{\mathrm{m}}-V_{\max }$ relationship, two barriers positioned in serial separated by a periplasm should produce a quadratic-like function, which equation (5) is. A power-law fit to empirical $\left(V_{\max }, K_{\mathrm{m}}\right)$ data of the form $K_{m} \approx a \cdot V_{\max }^{b}$ is consistent with equation (5) because $b \approx 1.86 \pm 0.23$ (mean \pm estimated s.e.m., degree of freedom $=8$, Fig. $3 \mathrm{~b}$ ). We note that the role played by MalE in E. coli is neglected as part of the derivation of equation (5) (in Supplementary Discussion, section B), but if we were to assume that MalE-maltotriose binding is so rapid that no free maltotriose is found in the periplasm, then the form of equation (5) will not change. 
To test whether the RATO could promote the maintenance of bacterial diversity, we isolated a subset of our library that exhibited a RATO. Three strains, labelled 9a (a predicted high-affinity strain; an AAC deletion at $871 \mathrm{bp}$ in $\operatorname{lamB}$ ), 19a (a frameshift mutant with nonfunctional LamB, $\mathrm{T}$ insertion at $610 \mathrm{bp}$ ) and $62 \mathrm{~b}$ (predicted low-affinity strain; $A \rightarrow G$ at $1,211 \mathrm{bp}$ ) were identified with different maltotriose transport properties (Fig. $3 b$ ), one-way analysis of variance, $F \approx 28.2, P<7 \times 10^{-13}$ ). The three-strain version of equation (3) predicts they should coexist in a fluctuating resource environment, such as a batch culture, where bacteria are inoculated into fresh flasks each day with high initial resources that are depleted throughout a $24-\mathrm{h}$ cycle as the bacteria grow (Fig. 4a).

We cultured 9a, 19a and $62 \mathrm{~b}$ in DM with $300 \mu \mathrm{g} \mathrm{ml}^{-1}$ of maltotriose for 18 days in such a batch culture microcosm and found that, consistent with predictions, all three genotypes persisted. However, the model is not quantitative (Fig. 4b): it fails to predict the relative frequencies at which each genotype was maintained. Most notably, the microcosm was dominated by the high-affinity strain, 9a, whereas the model predicts that the lowaffinity strain, 62b, should prevail (Fig. 4a). This shows that consumer lifestyle and the between-strain RATO alone are not sufficient to explain the dynamics we observe.

To understand why theory and experiments disagreed, we reasoned that the RYTO could play a role because it could lead fast-uptake genotypes to have low efficiencies which would impact growth rates. To justify this we followed the path of maltotriose through the cell which is converted to glucose and an excess of glucose produced at high maltotriose concentrations will lead glycolytic NADH to repress citrate synthase and, therefore, the TCA cycle ${ }^{29}$. As total ATP yield from the TCA cycle is greater per glucose supplied than from glycolysis, this repression should strengthen the within-strain RYTO we observed (Fig. 1). We therefore included a glucose-dependent, maltotriose-to-biomass conversion efficiency into the model to mimic the RYTO (Supplementary Discussion, section D). Only then, with two trade-offs, do we have a theory consistent with the 18-day mean frequency dynamics of the strains that also exhibits the long-term coexistence of all three (Fig. 4c).

To test for stable coexistence, we inoculated the three strains into a fresh microcosm at relative frequencies predicted by the trained dual trade-off model after running it to equilibrium. After so doing, we found no evidence of a deviation from the predicted strain frequencies when propagating them for further 7 days of seasonal co-culture (Fig. 4c and Supplementary Discussion, section $\mathrm{D})$. The eventual deviation that we observed of the microcosm from the theoretical prediction is only to be expected as the coexisting lineages likely gain further mutations that are not encoded within the model (Supplementary Discussion, section D).

\section{Discussion}

Trade-off shape is believed to be a key to understanding species diversity in global ecosystems and our analyses concur. Our theoretical models and empirical analyses elucidate the shape of two, the RATO and RYTO. According to our theory, both must be invoked simultaneously to explain the stable maintenance of genetic diversity in an E. coli transporter gene, $\operatorname{lamB}$, when mutants are co-cultured within a seasonal resource dynamic. The RATO, with its quadratic shape (Fig. 3b), is the result of a biophysical constraint that arises because the transport of extracellular nutrients into the cell is optimized. As different transporter structures are optimal for different extracellular nutrient concentrations, a continuum of potential sugar uptake niches is created (Fig. 4a) that here maintains three genetic lineages. However, nutrient consumption alone cannot account for strain frequencies and differential efficiencies resulting from downstream metabolism due to the RYTO, with its sigmoidal shape (Fig. 1c), ensure that efficient and not greedy consumers prevail.

Trade-offs have long been postulated as a mechanism supporting genetic diversity in ecosystems and it has become a fundamental problem in ecology to find them in living systems ${ }^{30}$. Importantly, we show they are predictable: because trade-offs are the result of physical processes within the cell, their shapes can be derived mathematically and corroborated against empirical data. Consistent with trade-off theories of biodiversity, we also show that polymorphisms in specific feeding genes are predictable. However, just one trade-off was not enough, we need to account for the simultaneous presence of two to correctly predict allele frequencies in a bacterial microcosm. Therefore, theories of multiple simultaneous trade-offs ${ }^{31}$ are likely needed to capture the diversity observed in natural ecosystems.

\section{Methods}

IamB mutant library generation. We co-cultured E. coli strain B(REL606), from the laboratory of Richard Lenski, Michigan State University, Michigan, USA, with the obligatorily lytic $\lambda$ strain cI26, from the laboratory of Donald Court, National Cancer Institute, Maryland, USA. When co-cultured with cI26, REL606 experiences strong pressure to evolve resistance because E. coli $B$ strains lack generalized phage defenses such as mucoid cell formation, restriction modification or CRISPR adaptive immunity ${ }^{32,33}$. This pressure is magnified by the lytic phage's increased virulence as compared with its lysogenic relatives ${ }^{34}$. About 100 flasks were initiated with $10^{3}$ bacterial cells and even fewer phage $\left(\sim 10^{2}\right.$ particles). We initiated the study with small populations to increase the likelihood that mutations for defense would arise de novo, which improved our chances of isolating unique $\operatorname{lamB}$ mutations. Culturing occurred in $50 \mathrm{ml}$ Erlenmeyer flasks, filled with $10 \mathrm{ml}$ of modified $\mathrm{DM}^{35}\left(125 \mathrm{\mu g} \mathrm{ml}^{-1}\right.$ maltotriose instead of glucose and $1 \mu \mathrm{g} \mathrm{ml}^{-1}$ of magnesium sulphate), incubated for $24 \mathrm{~h}$, at $37^{\circ} \mathrm{C}$ and shaken at 120 r.p.m. Bacteria were pre-conditioned for a full 24-h cycle before being co-cultured with phage. After the first $24 \mathrm{~h}$ of incubation in the mixed cultures, a random $100 \mu \mathrm{l}$ sample of each flask was transferred to a fresh flask and the bacteria and phage were allowed to grow again. This cycle was repeated twice and bacteria were sampled after the third day of growth. We ended the experiment at this early time-point to ensure that the bacteria only had enough time to evolve a single mutation for defense. In addition, in previous experiments we observed the greatest genotypic diversity of bacteria on the third day. E. coli evolve resistance in this environment through mutations in many loci, however, preliminary experiments revealed that lamB is the most common locus for $\lambda$ resistance mutations. For this study, we focused just on genotypes that had mutations in $\operatorname{lam} B$.

Bacterial isolation and storage. To isolate bacteria we streaked a sample on Luria Bertani (LB) agar plates ${ }^{36}$, incubated them for $24 \mathrm{~h}$ at $37^{\circ} \mathrm{C}$, and arbitrarily picked two colonies. Next, we re-plated each colony two more times serially to remove all phage. Finally, we grew each colony in liquid LB overnight and preserved two $1 \mathrm{ml}$ samples in $15 \%$ glycerol and froze it at $-80^{\circ} \mathrm{C}$. We tested whether each isolate had evolved resistance to $\lambda$ by spot assay ${ }^{37}$, where $2 \mu \mathrm{l}$ of the concentrated phage stock is dried on top of a matrix of $4 \mathrm{ml}$ of soft agar (LB agar with only $4 \% \mathrm{w} / \mathrm{v}$ agar) and $0.5 \mathrm{ml}$ of the LB overnight culture. The matrix is suspended on top of a typical LB agar Petri dish. Each plate was incubated at $37^{\circ} \mathrm{C}$ for $24 \mathrm{~h}$ and then scored the next day. A strain was considered resistant if no clearing occurred under the spot where the phage lysate was applied. We sequenced each of the resistant genotypes to determine if resistance had evolved through mutations in lamB.

Sequencing. Sequencing was performed with an automated ABI sequencer maintained at Michigan State University Research Technology Support Facility. PCR-amplified fragments that were purified with GFX columns and were used as templates. Fragments containing $\operatorname{lamB}$ were amplified with primer sequences $5^{\prime}$-TTCCCGGTAATGTGGAGATGC- $3^{\prime}$ and $5^{\prime}$-AATGTTTGCCGGGACGCT GTA-3', placed 1,398 bases up and 504 bases downstream of the lamB gene, respectively.

Growth dynamics. Each genotype was revived by inoculating a few microliters of frozen culture into a tube with $4 \mathrm{ml}$ of liquid LB. Cultures were grown at $37^{\circ} \mathrm{C}$ and shaken at 160 r.p.m. After $12 \mathrm{~h}, 10 \mu \mathrm{l}$ of each culture was transferred to a fresh tube with modified DM250 and grown for $12 \mathrm{~h}$ at $37^{\circ} \mathrm{C}$ and shaken at 160 r.p.m. This acclimation step to DM250 was repeated again because preliminary studies showed that growth was much more consistent when two sequential acclimation steps were performed. Finally, $2 \mu \mathrm{l}$ of each culture ( $10^{6}$ cells) was inoculated into a well with 
$200 \mu \mathrm{l}$ of modified DM. Wells were randomized in a 96-well plate, however, cultures were never inoculated into the outer-rim to avoid edge effects caused by increased rates of evaporation. Next, the cells were incubated at $37^{\circ} \mathrm{C}$ and the optical densities (at $420 \mathrm{~nm}$ wave length) of each well was measured once every $5 \mathrm{~min}$ for $12 \mathrm{~h}$ using a VersaMax automated plate reader (Molecular Devices). Each genotype was grown in modified DM supplemented with either $25 \mu \mathrm{g} \mathrm{ml}^{-1}$ of maltotriose or $250 \mu \mathrm{g} \mathrm{ml}^{-1}$, in four separate replicates. Optical densities (OD) was converted into cell densities by comparing counts of colony-forming units to ODs at a number of different cell concentrations. Growth curves were created identically for the ancestor, REL606, except at many more concentrations of maltotriose $(1,2$, $4,8,16,32,64$ and $128 \mu \mathrm{g} \mathrm{ml}^{-1}$ ). These data were used to measure the RYTO.

Coexistence experiments. Coexistence experiments were run under the same conditions as the growth dynamics were measured, except maltotriose concentrations were increased to $333 \mu \mathrm{g} \mathrm{ml}^{-1}$, plates were continuously shaken at 220 r.p.m. and the experiments were run for 18 days by transferring a random sample of $2 \%$ of the culture after $24 \mathrm{~h}$ of growth to a well with fresh medium. Coexistence was measured by tracking co-occurring populations of three genotypes-9a, 19a and 62b. To track the populations, we used the ara genetic marker. Each of the three lamB mutant genotypes were unable to metabolize $\mathrm{L}$-arabinose (ara-) because of a single nucleotide substitution in $\operatorname{araA}$ that caused a G-to-D amino acid change at position 92 . We selected for spontaneous reversions of this mutation by growing $\sim 10^{9}$ cells of each strain on minimal arabinose plates for $48 \mathrm{~h}$ at $37^{\circ} \mathrm{C}$ (ref. 35). Reversions were verified by targeted sequencing of ara $\mathrm{A}$ using the same protocol for lamB, except the following primers were used for PCR amplification: $5^{\prime}$-CCGATACGCTCATGGGCTTGTTTA- $3^{\prime}$ and $5^{\prime}$-CTGCCCAGG CCGTTGCGACTCTAT- $3^{\prime}$. The ara genetic marker was chosen because previous work has shown that it does not confer a $\operatorname{cost}^{35}$. We cultured each ara + genotype with the ara - version of the other two lamB mutants. The density of the focal genotype was measured by counting colonies on minimal arabinose plates. Total bacterial population size was also measured by counting colonies on LB plates. For each ara + genotype, we constructed four replicate populations making 12 coexistence experiments in total.

\section{References}

1. Maharjan, R. et al. The form of a trade-off determines the response to competition. Ecol. Lett. 16, 1267-1276 (2013).

2. Ben-Hur, E., Fragman-Sapir, O., Hadas, R., Singer, A. \& Kadmon, R. Functional trade-offs increase species diversity in experimental plant communities. Ecol. Lett. 15, 1276-1282 (2012).

3. Kraaijeveld, A. R. \& Godfray, H. C. Trade-off between parasitoid resistance and larval competitive ability in drosophila melanogaster. Nature 389, 278-280 (1997).

4. Fine, P. V. A. et al. The growth-defense trade-off and habitat specialization by plants in amazonian forests. Ecology 87, S150-S162 (2006).

5. Leiby, N. \& Marx, C. J. Metabolic erosion primarily through mutation accumulation, and not tradeoffs, drives limited evolution of substrate specificity in Escherichia coli. PLoS Biol. 12, e1001789 (2014).

6. Friesen, M. L., Saxer, G., Travisano, M. \& Doebeli, M. Experimental evidence for sympatric ecological diversification due to frequency-dependent competition in Escherichia coli. Evolution 58, 245-260 (2004).

7. Poelwijk, F. J., de Vos, M. G. J. \& Tans, S. J. Tradeoffs and optimality in the evolution of gene regulation. Cell 146, 462-470 (2011).

8. Herron, M. D. \& Doebeli, M. Parallel evolutionary dynamics of adaptive diversification in Escherichia coli. PLoS Biol. 11, e1001490 (2013).

9. Fitzsimmons, J., Schoustra, S., Kerr, J. \& Kassen, R. Population consequences of mutational events: effects of antibiotic resistance on the R/K trade-off. Evol. Ecol. 24, 227-236 (2010).

10. Wong, W. W., Tran, L. M. \& Liao, J. C. A hidden square-root boundary between growth rate and biomass yield. Biotechnol. Bioeng. 102, 73-80 (2009).

11. MacLean, R. C. \& Gudelj, I. Resource competition and social conflict in experimental populations of yeast. Nature 411, 498-501 (2006).

12. Novak, M., Pfeiffer, T., Lenski, R. E., Sauer, U. \& Bonhoeffer, S. Experimental tests for an evolutionary trade-off between growth rate and yield in E. coli. Am. Nat. 168, 242-251 (2006).

13. Pfeiffer, T., Schuster, S. \& Bonhoeffer, S. Cooperation and competition in the evolution of ATP-producing pathways. Science 292, 504-507 (2001).

14. Gudelj, I. et al. An integrative approach to understanding microbial diversity: from intracellular mechanisms to community structure. Ecol. Lett. 13, 1073-1084 (2010).

15. Smith, S. L., Pahlow, M., Merico, A. \& Wirtz, K. W. Optimality-based modeling of planktonic organisms. Limnol. Oceanogr. 56, 2080-2094 (2011).

16. Stewart, F. M. \& Levin, B. R. Partitioning of resources and the outcome of interspecific competition: a model and some general considerations. Am. Nat. 107, 171-198 (1973).
17. Dethlefsen, L. \& Schmidt, T. M. Performance of the translational apparatus varies with the ecological strategies of bacteria. J. Bacteriol. 189, 3237-3245 (2007).

18. MacArthur, R. \& Wilson, E. The Theory of Island Biogeography (Princeton Univ. Press, 1967).

19. Boos, W. \& Shuman, H. Maltose/maltodextrin system of Escherichia coli: transport, metabolism, and regulation. Microbiol. Mol. Biol. Rev. 62, 204-229 (1998).

20. MacLean, R. C. \& Gudelj, I. Resource competition and social conflict in experimental populations of yeast. Nature 441, 498-501 (2006).

21. Nikaido, H. \& Vaara, M. Molecular basis of bacterial outer membrane permeability. Microbiol. Rev. 49, 1-32 (1985).

22. Saint, N. et al. Structural and functional characterization of ompf porin mutants selected for larger pore size. ii. functional characterization. J. Biol. Chem. 271, 20676-20680 (1996).

23. Thirion, J. P. \& Hofnung, M. On some genetic aspects of phage lambda resistance in E. coli k12. Genetics 71, 207-216 (1972).

24. Braun-Breton, C. \& Hofnung, M. In vivo and in vitro functional alterations of the bacteriophage lambda receptor in lamb missense mutants of escherichia coli k-12. J. Bacteriol. 148, 845-852 (1981).

25. Heine, H. G., Kyngdon, J. \& Ferenci, T. Sequence determinants in the lamb gene of escherichia coli influencing the binding and pore selectivity of maltoporin. Gene 53, 287-292 (1987).

26. Elbing, K. et al. Role of hexose transport in control of glycolytic flux in Saccharomyces cerevisiae. Appl. Environ. Microbiol. 70, 5323-5330 (2004)

27. Berezhkovskii, A. M. \& Bezrukov, S. M. Optimizing transport of metabolites through large channels: molecular sieves with and without binding. Biophys. J. 88, L17-L19 (2005).

28. Pagliara, S., Schwall, C. \& Keyser, U. F. Optimizing diffusive transport through a synthetic membrane channel. Adv. Mater. 25, 844-849 (2013).

29. Stulke, J. \& Hillen, W. Carbon catabolite repression in bacteria. Curr. Opin. Microbiol. 2, 195-201 (1999).

30. Agrawal, A. A., Conner, J. K. \& Rasmann, S. in Evolution After Darwin: the First 150 Years. (eds Bell, M.A., Eanes, W.F. \& Futuyma, D.J.) (Sinauer Associates, 2010).

31. Doebeli, M. \& Ispolatov, I. Complexity and diversity. Science 328, 494-497 (2010).

32. Spanakis, E. \& Horne, M. T. Co-adaptation of Escherichia coli and coliphage lambda vir in continuous culture. J. Gen. Microbiol. 133, 353-360 (1987).

33. Daegelen, P., Studier, F. W., Lenski, R. E., Cure, S. \& Kim, J. F. Tracing ancestors and relatives of escherichia coli $\mathrm{b}$, and the derivation of $\mathrm{b}$ strains rel606 and bl21(de3). J. Mol. Biol. 394, 634-643 (2009).

34. Lenski, R. E. in Advances in Microbial Ecology. (ed. Marshall, K. C.) Vol. 10 1-44 (Plenum Press, 1988).

35. Lenski, R., Rose, M., Simpson, S. \& Tadler, S. Long-term evolution experiment in Escherichia coli 1. adaptation and divergence during 2,000 generations. Am. Nat. 138, 1315-1341 (1991).

36. Sambrook, J. \& Russell, D. W. Molecular Cloning: a Laboratory Manual 3rd edn (Cold Spring Harbor Laboratory Press, 2001).

37. Adams, M. Bacteriophages (Interscience Publishers, 1959).

38. Charbit, A. et al. In vivo and in vitro studies of transmembrane beta-strand deletion, insertion or substitution mutants of the Escherichia coli K-12 maltoporin. Mol. Microbiol. 35, 777-790 (2000).

\section{Acknowledgements}

We thank Chris Marx, Tom Ferenci, Christina Burch and other members of a NESCent working group 'Mathematical Models, Microbes and Evolutionary Diversification' for helpful discussions. We thank Richard Lenski and Donald Court for supplying bacteria and phage.

\section{Author contributions}

Experiments were performed by J.R.M., theoretical calculations were performed by R.B. and I.G., all authors wrote the manuscript.

\section{Additional information}

Supplementary Information accompanies this paper at http://www.nature.com/ naturecommunications

Competing financial interests: The authors declare no competing financial interests.

Reprints and permission information is available online at http://npg.nature.com/ reprintsandpermissions/

How to cite this article: Meyer, J. R. et al. Biophysical mechanisms that maintain biodiversity through trade-offs. Nat. Commun. 6:6278 doi: 10.1038/ncomms7278 (2015). 